Combining hyperpolarized real-time metabolic imaging and NMR spectroscopy to identify metabolic biomarkers in pancreatic cancer

Prasanta Dutta ${ }^{1}$, Mayrim R. Perez ${ }^{2}$, Jaehyuk Lee ${ }^{1}$, Yaan Kang ${ }^{2}$, Michael Pratt ${ }^{2}$, Travis C. Salzillo ${ }^{1}$, Joseph Weygand $^{1}$, Niki M. Zacharias ${ }^{1,3}$, Seth T. Gammon ${ }^{1}$, Eugene J. Koay ${ }^{3}$, Michael Kim², Florencia McAllister ${ }^{4}$, Subrata Sen ${ }^{5}$, Anirban Maitra ${ }^{6}$, David Piwnica-Worms ${ }^{1}$, Jason B. Fleming ${ }^{2,7}$, Pratip K. Bhattacharya ${ }^{1 *}$

${ }^{1}$ Department of Cancer Systems Imaging, The University of Texas MD Anderson Cancer Center, Houston, Texas, USA

${ }^{2}$ Department of Surgical Oncology, The University of Texas MD Anderson Cancer Center, Houston, Texas, USA ${ }^{3}$ Department of Radiation Oncology, The University of Texas MD Anderson Cancer Center, Houston, Texas, USA ${ }^{4}$ Department of Clinical Cancer Prevention, The University of Texas MD Anderson Cancer Center, Houston, Texas, USA

${ }^{5}$ Department of Translational Molecular Pathology, The University of Texas MD Anderson Cancer Center, Houston, Texas, USA

${ }^{6}$ Department of Pathology, The University of Texas MD Anderson Cancer Center, Houston, Texas, USA

${ }^{7}$ Department of Gastrointestinal Oncology, H. Lee Moffitt Cancer Center, Tampa, Florida, USA

*To whom correspondence should be addressed:

Email: PKBhattacharya@mdanderson.org

Supporting Figure S1: The real-time metabolic ${ }^{13} \mathrm{C}$ MRS in vivo after injecting hyperpolarized pyruvate intravenously in (A) most aggressive tumor MDA-PATX148, (B) less aggressive tumor MDA-PATX141 and (C) least aggressive tumor MDA-PATX142.

Supporting Figure S2: ${ }^{1} \mathrm{H}$ NMR high resolution spectra are showing different metabolites peak intensities for all four tumor types. 
Figure S1. (A) Dynamic Hyperpolarized ${ }^{13} \mathrm{C}$ MRS In Vivo (Tumor: PATX148; most aggressive)

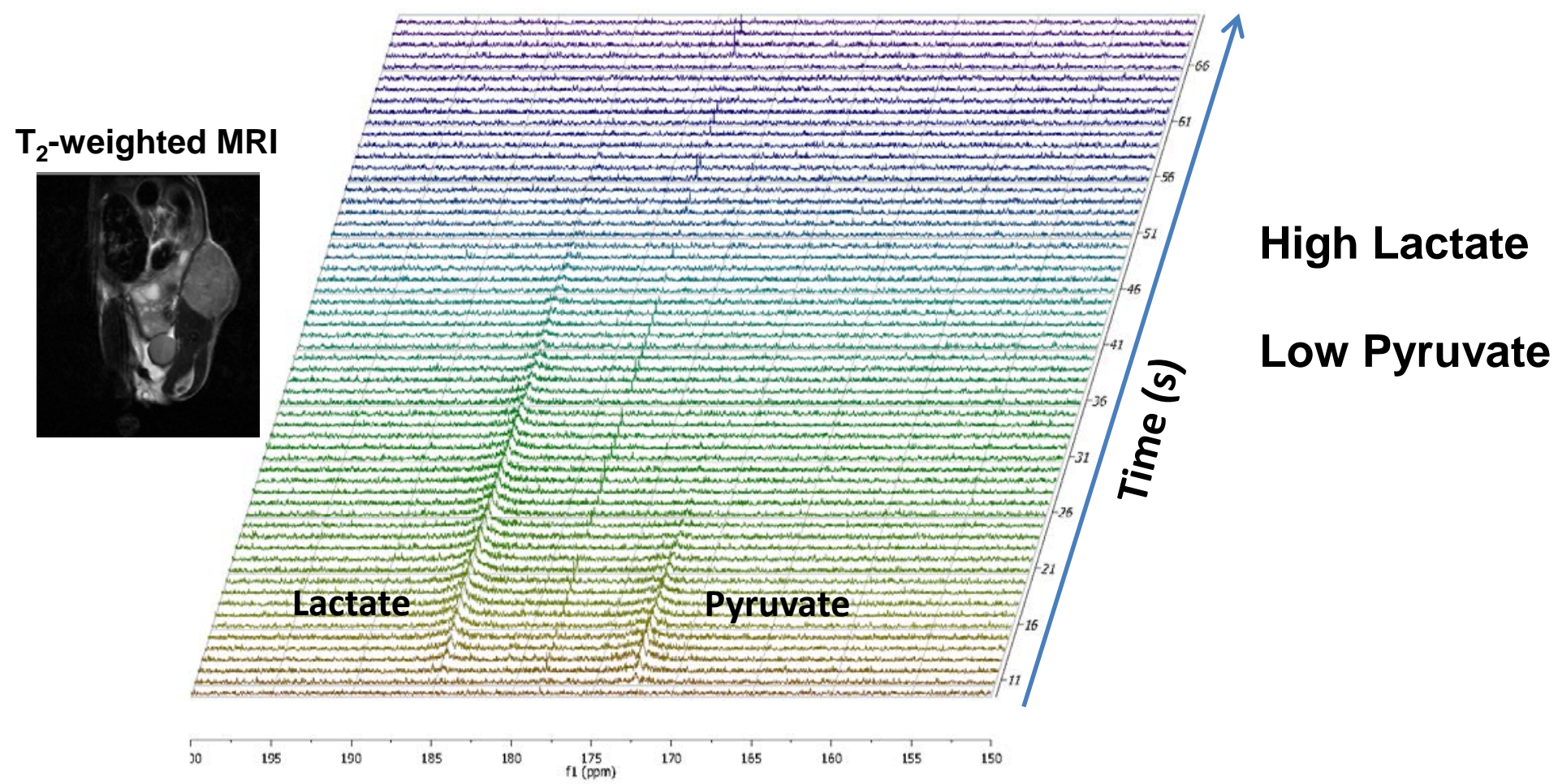


Figure S1. (B) Dynamic Hyperpolarized ${ }^{13} \mathrm{C}$ MRS In Vivo (Tumor: PATX-141)

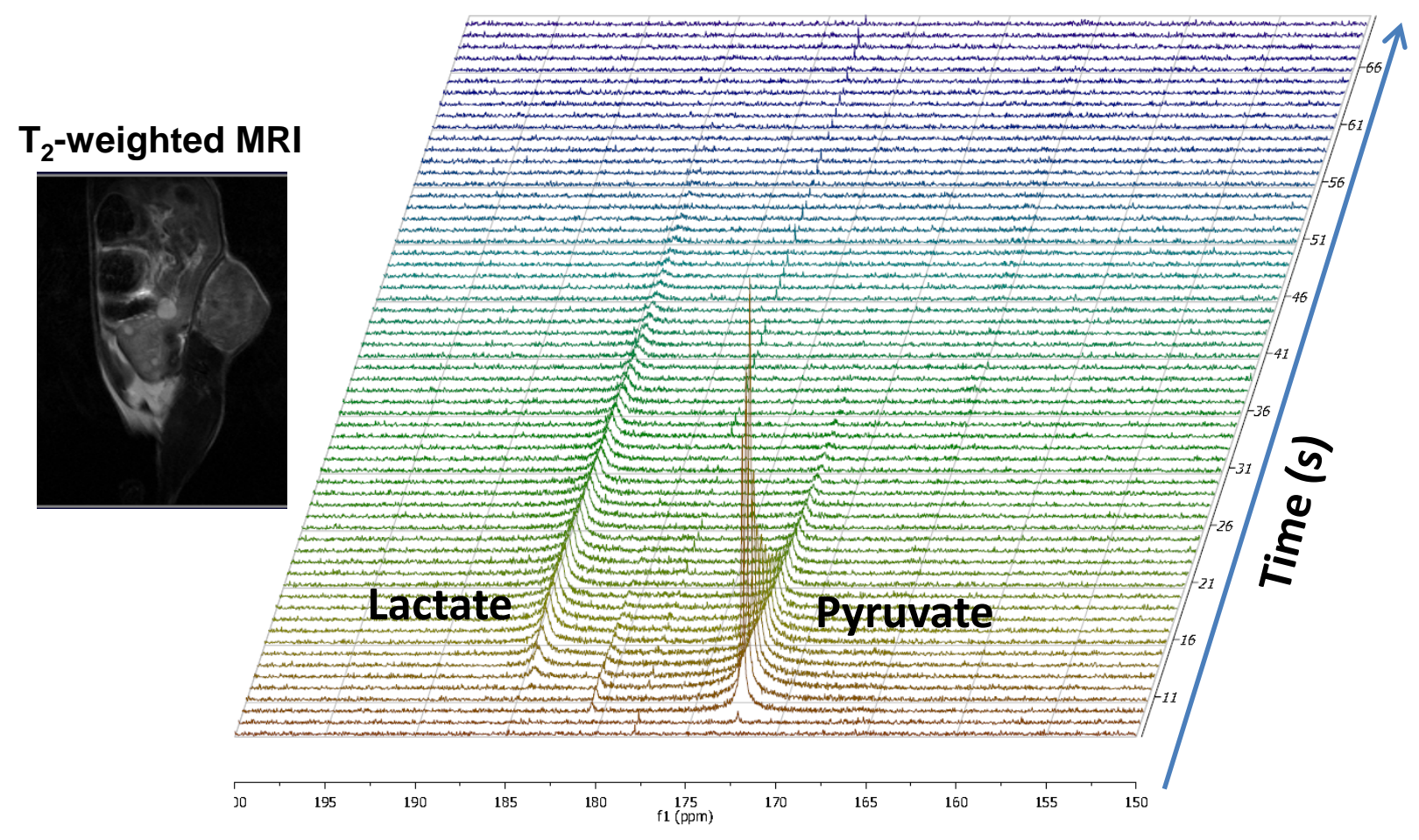


Figure S1. (C) Dynamic Hyperpolarized ${ }^{13} \mathrm{C}$ MRS In Vivo (Tumor: PATX-142; least aggressive))

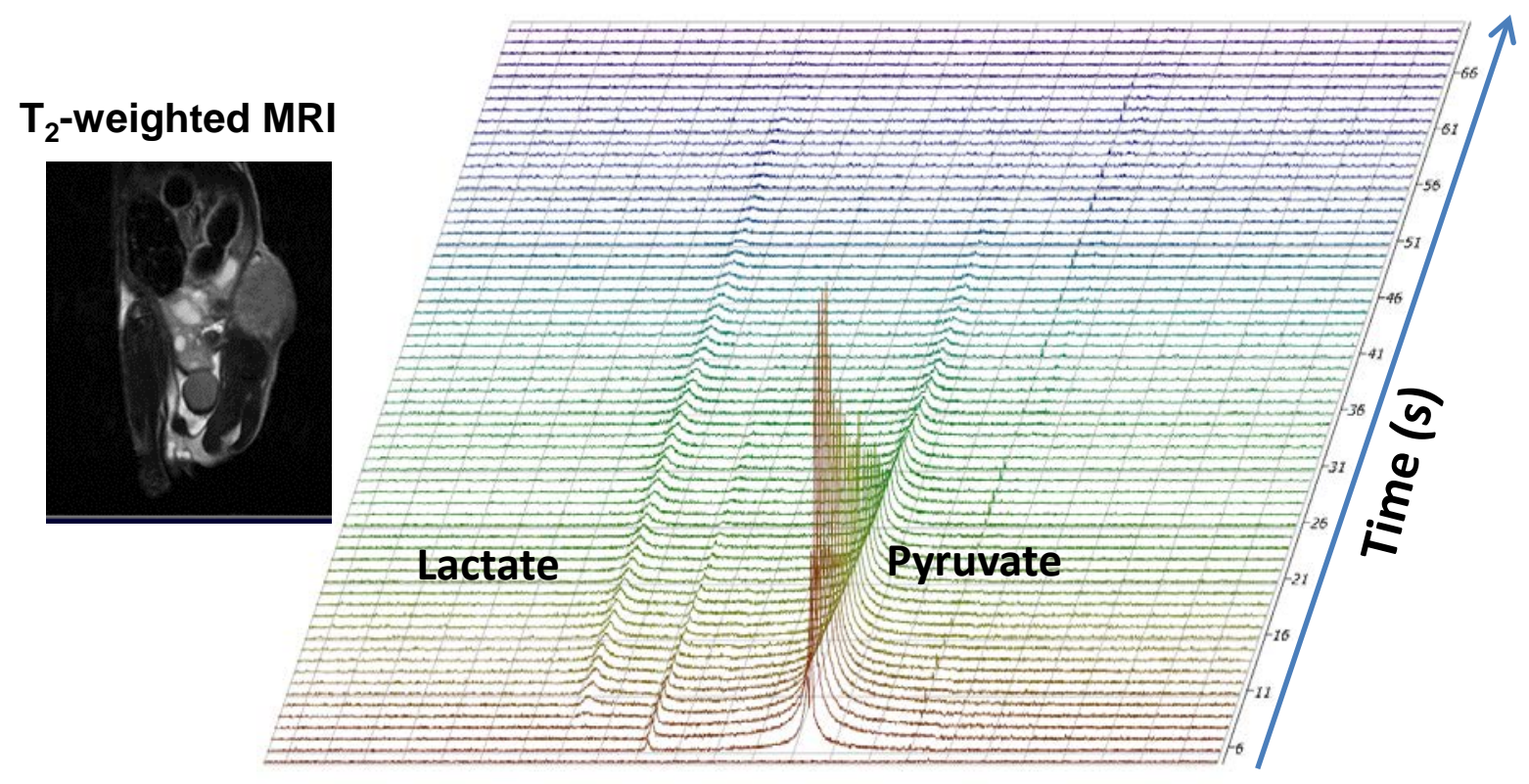

High Pyruvate

Low Lactate

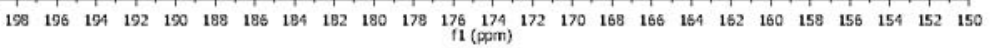


Figure S2. ${ }^{1} \mathrm{H}$ NMR metabolomics for four different tumor types ex vivo.

PC: Phosphocholine

GPC: Glycerophosphocholine
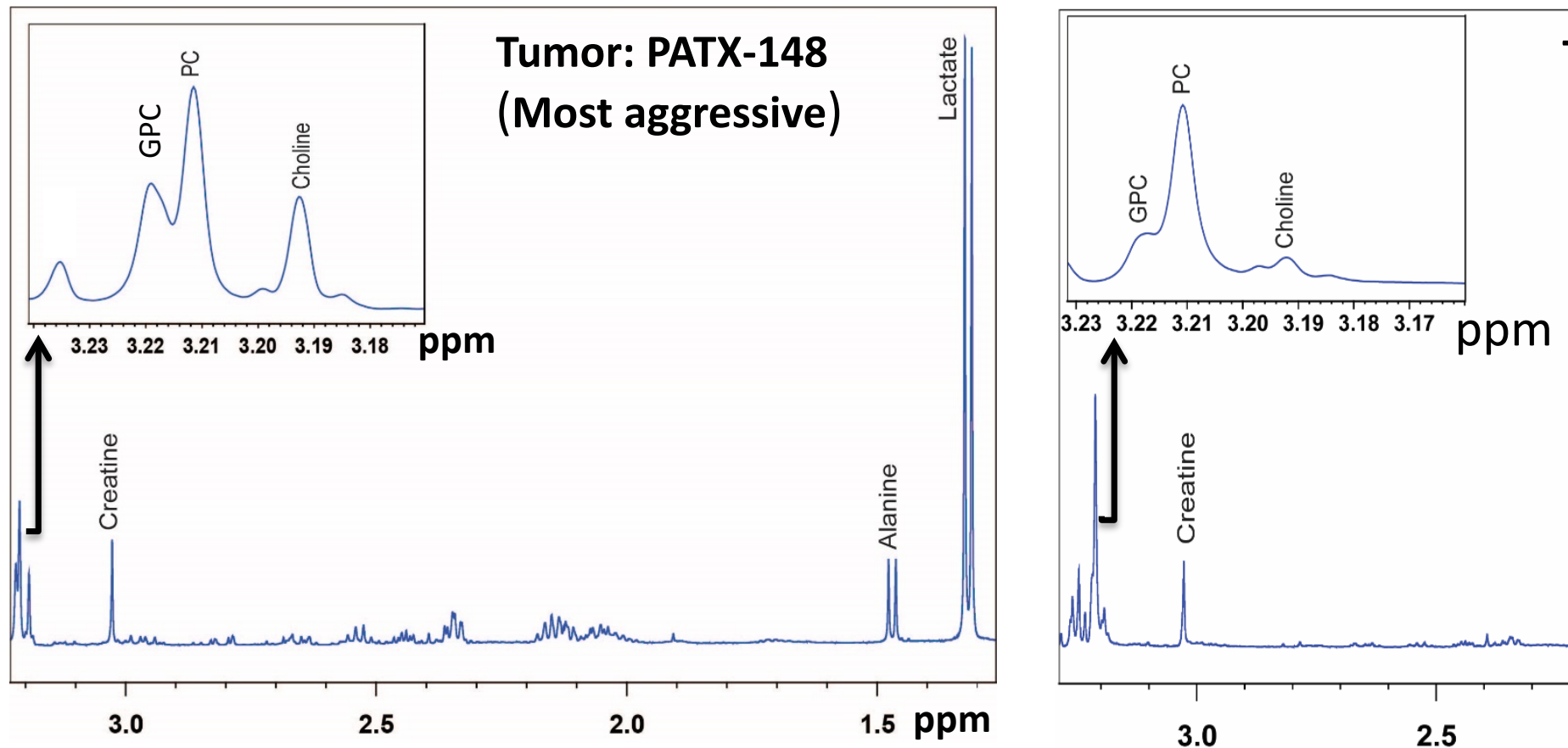

\section{Tumor: PATX-127}
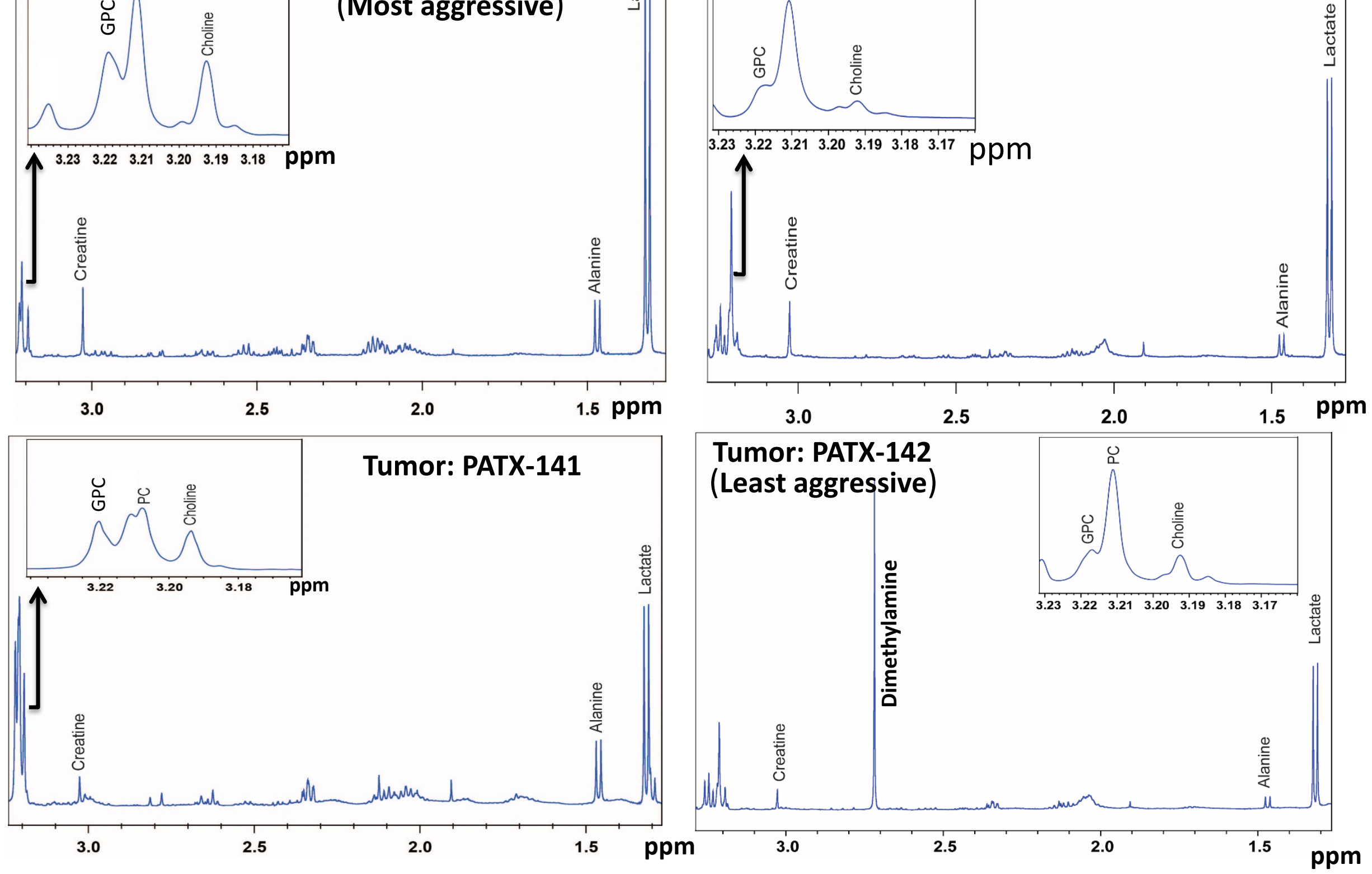